\title{
Kinematics of superdense galaxies in clusters
}

\author{
Alessia Moretti ${ }^{1}$, Bianca Poggianti ${ }^{2}$, Daniela Bettoni ${ }^{2}$, \\ Michele Cappellari ${ }^{3}$, Giovanni Fasano ${ }^{2}$ and the WINGS team \\ ${ }^{1}$ Department of Physics and Astronomy, University of Padova, \\ Vicolo dell'osservatorio 3, 35127 Padova, Italy \\ email: alessia.moretti@unipd.it \\ ${ }^{2}$ INAF - Padova Astronomical Observatory \\ Vicolo dell'osservatorio 2, 35127 Padova, Italy \\ ${ }^{3}$ Dep. of Astrophysics, University of Oxford, Denys Wilkinson Building, Keble Road, Oxford, \\ OX1 3RH, UK
}

\begin{abstract}
We present the first results obtained by analyzing the detailed kinematics of a subsample of 9 massive and compact galaxies found in the WINGS survey. The observed galaxies are very old (both luminosity and mass- weighted age are on average $\geqslant 10 \mathrm{Gyr}$ ), while they resemble more typical galaxies in the other characteristics. The total $\mathrm{M} / \mathrm{L}$ ratio is determined using as free parameters the anisotropy $\beta$ and the galaxy inclination $i$.
\end{abstract}

Keywords. galaxies: elliptical and lenticular, cD - galaxies: evolution - galaxies: formation

\section{Sample selection and analysis}

Valentinuzzi et al., 2010 found in the WINGS sample of local cluster of galaxies $(0.04<z<0.07)$ a significant number of massive and compact galaxies $\left(3 \times 10^{10} M_{\odot}<\right.$ $\left.M<4 \times 10^{11} M_{\odot}, \Sigma_{50}>3 \times 10^{9} M_{\odot} / k p c^{2}\right)$, that have not been found in other local surveys (e.g. SDSS). Our results show that selecting already passive galaxies at high $\mathrm{z}$ means selecting the most compact ones. Assuming they do not resume the star formation activity at later times, the high-z sizes should be compared with the sizes of the oldest (in terms of their LW-age) low-z galaxies, to avoid a significant progenitor bias. Taking into account this progenitor bias Poggianti et al., 2013b found that for compact galaxies in low density environments a significant size evolution for these compact objects is not needed. In order to validate our stellar masses, derived using fiber spectroscopy and our spectrophotometric tool SINOPSIS (Fritz et al., 2007,2011), we obtained two sets of IFU observations for a subsample of 17 WINGS SDGs. 9 of them have been observed with G-MOS @GEMINI and 8 of them with VIMOS (purple and green dots, respectively, in Fig. 1, top left panel).

We present here WINGS SDGs that have been observed with GEMINI G-MOS with an exposure time of $2700 \mathrm{~s}$, using the B600 grism in the 1-slit mode. The FoV is $3.5^{\prime \prime} \times 5^{\prime \prime}$. The wavelength coverage goes from 4000 to $6900 \AA$. We derived the stellar kinematics by using the pPXF algorithm by Cappellari \& Emsellem (2004), that works in regions where $\mathrm{S} / \mathrm{N}=10$ (defined by a Voronoi tessellation). In our case we used as templates a set of MILES SSPs with 16 values of ages and 6 metallicities. Fig. 1, bottom panel shows the velocity map for one of our galaxies. We derived the luminosity-weighted angular momentum $\lambda_{R}$ following Emsellem et al., 2007, and found that most SDGs are fast rotators (Fig. 1, top right panel). We then started evaluating the dynamical mass using the Jeans Anysotropic MGE models by Cappellari 2008. This model requires an accurate estimate of the galaxy light decomposition, that is made by superimposing a set of multiple gaussian profiles (MGE expansion). We then used a self-consistent JAM 

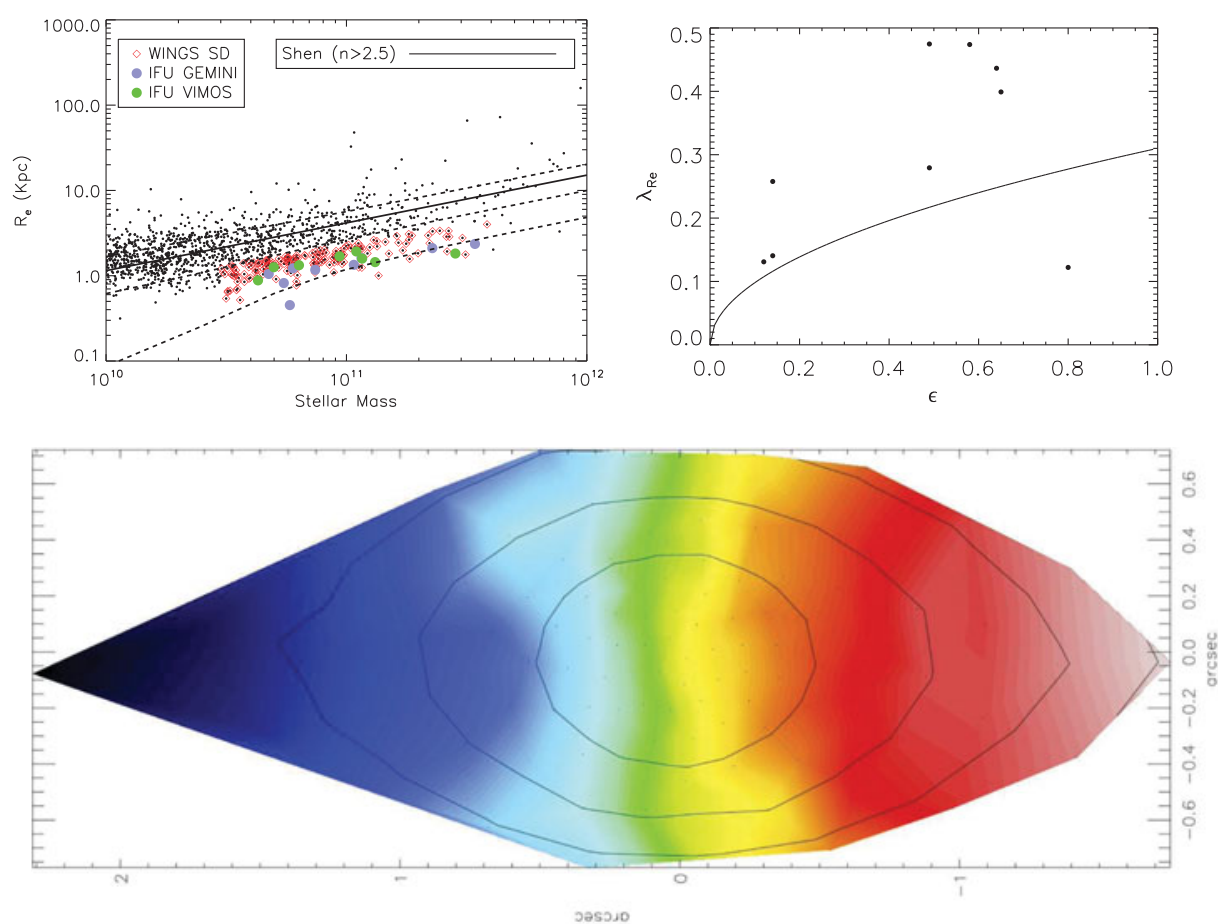

Figure 1. Top left: Mass-size relation for WINGS cluster members. Black lines are the local (SDSS) relations. Red dots are WINGS SDGs. Green and purple symbols are IFU candidates. Top right: Luminosity weighted angular momentum $\left(\lambda_{R}\right)$ within $\mathrm{R}_{e}$ versus ellipticity. The line divides fast rotators (FR) from slow rotators (SR). Bottom panel: example of the velocity map derived for our SDGs.

model (see Cappellari et al., 2013, Paper XV), where the underlying assumption is that the mass distribution follows the light one derived from the MGE fit, to evaluate the M/L ratio. This model has two non linear parameters (the vertical anisotropy $\beta$ and the galaxy inclination $i$ ). The $\mathrm{M} / \mathrm{L}$ ratio is then derived imposing the match with the observed $V_{r m s}$. The derived M/L is the total one, and not only the stellar one. We are currently deriving the M/L JAM for our galaxies with the aim of estimating the dark matter fraction inside $R_{e}$ (by comparing it with the stellar $\mathrm{M} / \mathrm{L}$ derived from spectrophotometry) and the possible influence of the adopted IMF.

\section{References}

Cappellari M., 2008, MNRAS, 390, 71

Cappellari M., Emsellem E., 2004, PASP, 116, 138

Cappellari M., et al., 2013, MNRAS, 432, 1709

Emsellem E., et al., 2007, MNRAS, 379, 401

Fritz J., et al., 2007, A\&SA, 470, 137

Fritz J., et al., 2011, A\& $A, 526, \mathrm{~A} 45$

Poggianti B. M., et al., 2013, ApJ, 777, 125

Valentinuzzi T., et al., 2010, ApJ, 712, 226 